\title{
The effect of movement behavior on population density in patchy landscapes
}

\author{
Nazanin Zaker • Laurence Ketchemen • \\ Frithjof Lutscher
}

Received: date / Accepted: date

\begin{abstract}
Many biological populations reside in increasingly fragmented landscapes, where habitat quality may change abruptly in space. Individuals adjust their movement behavior to local habitat quality and show preferences for some habitat types over others. Several recent publications explore how such individual movement behavior affects population-level dynamics in a framework of reactiondiffusion systems that are coupled through discontinuous boundary conditions. While most of those works are based on linear analysis, we study positive steady states of the nonlinear equations. We prove existence, uniqueness and global stability, and we classify their qualitative shape depending on movement behavior. We apply our results to study the question why and under which conditions the total population density at steady state may exceed the total carrying capacity of the landscape.
\end{abstract}

Keywords Reaction-diffusion system · Interface conditions · Steady state · Population dynamics

Mathematics Subject Classification (2010) 35K37 · 35Q92 · 92D40

FL is grateful for funding from the Natural Sciences and Engineering Research Council of Canada for funding through the Discovery Grant program (RGPIN-2016-04795) and for a Discovery Accelerator Supplement (RGPAS-2016-492872)

N. Zaker

Department of Mathematics and Statistics

University of Ottawa

E-mail: nzake042@uottawa.ca

L. Ketchemen

Department of Mathematics and Statistics

University of Ottawa

E-mail: lketc034@uottawa.ca

F. Lutscher

Department of Mathematics and Statistics, and Department of Biology

University of Ottawa

E-mail: flutsche@uottawa.ca 


\section{Introduction}

The study of spatial population dynamics relates to some of the most fundamental questions in theoretical ecology: Why are individuals where they are? How does landscape structure affect population distribution in space? What effect does movement in response to habitat variation have on population density? These questions are of increasing importance as natural events and human activities create increasingly fragmented landscapes in which some biological populations may thrive while others struggle to survive.

Reaction-diffusion equations are a common and highly versatile tool to study such questions (Cantrell and Cosner, 2003). One fairly basic model for the density $u(x, t)$ of a species at location $x$ and time $t$ is the diffusive logistic equation

$$
\frac{\partial u}{\partial t}=D \frac{\partial^{2} u}{\partial x^{2}}+r(x) u\left(1-\frac{u}{K(x)}\right)
$$

Here, the diffusion coefficient $D$ measures the mean squared displacement of organisms under random motion, $r$ is the low-density growth rate, and $K$ denotes the local carrying capacity. With spatially constant parameters, this equation is widely used in applications (Andow et al., 1990). For heterogeneous landscapes, large amounts of data are required to estimate the spatially varying parameter functions $r(\cdot)$ and $K(\cdot)$. In addition, when landscape quality varies in space, the assumption of a spatially constant random diffusion process is unrealistic. Most organisms adapt their movement to habitat quality and show bias towards better habitats (Maciel and Lutscher, 2013; Crone et al., 2019). Spatially varying movement behavior can be included in (1) in a number of different ways, such as a Fickian diffusion term $\partial_{x}\left(D(x) \partial_{x} u\right)$ or an "ecological" diffusion term $\partial_{x}^{2}(D(x) u)$ (Turchin, 1998). The two cases show different behavior in simulations, but analytical results remain relatively abstract (e.g., existence of solutions, monotonicity).

A closely related but somewhat different approach to population dynamics in spatially varying landscapes is to consider landscapes that consist of "patches": regions in space that are homogeneous within but different from their neighbouring patches. One then formulates a reaction-diffusion equation with constant coefficients on each patch, and connects the equations for adjacent patches by matching conditions for the density and flux. This modelling approach was pioneered by Pacala and Roughgarden (1982) for two patches and by Shigesada et al. (1986) for infinite landscapes, and continued by Freedman et al. (1989); Cruywagen et al. (1996); Lutscher et al. (2006) and others. A major improvement on these earlier models occurred when Maciel and Lutscher (2013) introduced novel interface matching conditions, based on the work by Ovaskainen and Cornell (2003). These matching conditions not only allow us to include patch preference data, which are frequently collected in the field, into reaction-diffusion models, they also remove some biologically unrealistic behavior that the early models showed; see Maciel and Lutscher (2013) for a thorough discussion of this point. A number of recent studies use this new framework to study questions of persistence and spread (Maciel and Lutscher, 2015; Alqawasmeh and Lutscher, 2019b) and apply it to marine reserve design (Langebrake et al., 2011; Alqawasmeh and Lutscher, 2019a). However, most of their results are based on either linear analysis or numerical simulation (but see Maciel et al. (2019)). 
In the current work, we study the various aspects of a positive steady state of the nonlinear equations. More specifically, we prove the existence, uniqueness, and global stability of such a state, and we classify the possible qualitative behaviors of this state, depending on model parameters. We also apply our results to the question of whether and how the total population density at steady state could exceed the total carrying capacity of a landscape, depending on movement behavior. This latter question has puzzled theoretical ecologists since the discovery of the phenomenon by Freedman and Waltman (1977); see Zhang et al. (2017) for a recent review on the subject. While the original discovery emerged in a spatially implicit patch model, formulated as ordinary differential equations, the phenomenon can also be observed in the spatially explicit reaction-diffusion model (1). More precisely, if we denote by $u^{*}$ the steady-state solution of (1), then Lou (2006) proved that

$$
\int_{\Omega}\left[u^{*}(x)-K(x)\right] \mathrm{d} x>0
$$

for all $D>0$, in the special case of nonconstant functions $r(x)=K(x)$. DeAngelis et al. (2016) extended this result by showing that the same inequality holds when $r(\cdot)$ and $K(\cdot)$ are positively correlated and $D$ is small. Such a result is surprising at first because it says that the "carrying capacity" is not necessarily the upper limit of the population density on a landscape. It is also relevant when studying competition of two species and the question of (mutual) invasion of one by the other. We will obtain some sufficient conditions for when the corresponding inequality holds in our patch model, and we will show that in certain limiting cases, these conditions are also necessary. But we will also show that certain types of movement behavior will ensure that the corresponding inequality cannot hold.

We begin with a detailed description of our model and some preliminary results in Section 2. The existence and uniqueness proof, as well as global stability, is given in Section 3. Illustrations of our results, the classification of steady-state profiles and the relation between the total steady-state density and the total carrying capacity are given by Section 4 . We close with a discussion.

\section{Model presentation}

We consider the simplest scenario of a patchy one-dimensional landscape, consisting of two adjacent patches that are homogeneous within but differ from one another. We denote these patches by $\Omega_{1}=\left[-L_{1}, 0\right]$ and $\Omega_{2}=\left[0, L_{2}\right]$, respectively, and the population density on patch $i$ by $u_{i}(x, t)$. We refer to the point $x=0$ where the two patches meet as the interface and to $x=-L_{1}$ and $x=L_{2}$ as the boundary of our landscape. On each patch, individual movement and population dynamics are described by a reaction-diffusion equation. Hence, the equations for the densities on patch $i$ have the form

$$
\frac{\partial u_{i}(x, t)}{\partial t}=D_{i} \frac{\partial^{2} u_{i}(x, t)}{\partial x^{2}}+u_{i}(x, t) f_{i}\left(u_{i}(x, t)\right), \quad x \in \Omega_{i}, \quad i=1,2,
$$

where $D_{i}$ is the diffusion coefficient in patch $i$. In general, we assume that the per capita growth functions $f_{i} \in C^{2}[0, \infty)$ satisfy the conditions (see, e.g., Freedman (1980))

$$
f_{i}(0)>0 ; f_{i}^{\prime}\left(u_{i}\right)<0 ; \text { there exists } K_{i}>0 \text { such that } f_{i}\left(K_{i}\right)=0 .
$$


In biological terms, the per capita growth rate decreases with population density because of intraspecific competition. The maximum per capita growth rate $r_{i}=$ $f_{i}(0)$ occurs at low density. This assumption excludes an Allee effect, where the maximum per capita growth rate occurs at intermediate density (Courchamp et al., 2008). Parameter $K_{i}$ can be interpreted as the carrying capacity of patch $i$. For all explicit calculations and simulations, we will use the logistic growth function

$$
f_{i}(u)=r_{i}\left(1-\frac{u}{K_{i}}\right) .
$$

We assume that no individuals cross the boundaries of the two-patch landscape. Hence, we impose Neumann (no-flux) boundary conditions at $x=-L_{1}$ and $x=$ $L_{2}$, i.e.,

$$
\frac{\partial u_{1}\left(-L_{1}, t\right)}{\partial x}=\frac{\partial u_{2}\left(L_{2}, t\right)}{\partial x}=0, \quad t \geqslant 0 .
$$

With these conditions, our system can also be viewed as half a period of an infinite periodic landscape with alternating patches of lengths $2 L_{1}$ and $2 L_{2}$, respectively (Maciel et al., 2019).

At the interface between patches, i.e., at $x=0$, we impose the matching conditions for population density and flux that were derived from a random-walk model by Ovaskainen and Cornell (2003) and studied further by Maciel and Lutscher (2013). There are two conditions. One of them states that the population flux is continuous across the interface. This implies that no individuals are lost or gained from moving across the interface. Mathematically, continuity of the flux at the interface is expressed as

$$
D_{1} \frac{\partial u_{1}(0, t)}{\partial x}=D_{2} \frac{\partial u_{2}(0, t)}{\partial x}, \quad t \geqslant 0 .
$$

The other condition relates the densities on the two sides of the interface. To understand this condition, we denote by $p_{1}$ and $p_{2}$ the probabilities that an individual at the interface moves to patch 1 and patch 2 , respectively. We assume that individuals cannot stay at the interface, so that $p_{1}+p_{2}=1$. We also refer to $p_{i}$ as habitat preference. With this notation, the second interface condition reads (Ovaskainen and Cornell, 2003; Maciel and Lutscher, 2013)

$$
u_{1}(0, t)=k u_{2}(0, t), \quad k=\frac{p_{1}}{p_{2}} \frac{D_{2}}{D_{1}}, \quad t \geqslant 0 .
$$

Putting all the ingredients together, we have the following system of equations:

$$
\begin{cases}\frac{\partial u_{i}(x, t)}{\partial t}=D_{i} \frac{\partial^{2} u_{i}(x, t)}{\partial x^{2}}+u_{i}(x, t) f_{i}\left(u_{i}(x, t)\right), & (x, t) \in \Omega_{i} \times[0, \infty) ; \\ D_{1} \frac{\partial u_{1}(0, t)}{\partial x}=D_{2} \frac{\partial u_{2}(0, t)}{\partial x}, & t \geq 0 ; \\ u_{1}(0, t)=k u_{2}(0, t), & t \geq 0 ; \\ \frac{\partial u_{1}\left(-L_{1}, t\right)}{\partial x}=\frac{\partial u_{2}\left(L_{2}, t\right)}{\partial x}=0, & t \geq 0 .\end{cases}
$$


Uniqueness and global existence of solutions of this time-dependent problem were recently proved by Maciel et al. (2019). In this work, we focus on the steadystate problem of equations (9). Denoting the steady state densities also by $u_{i}=$ $u_{i}(x)$, the defining equations are

$$
\left\{\begin{array}{l}
D_{i} \frac{\mathrm{d}^{2} u_{i}(x)}{\mathrm{d} x^{2}}+u_{i}(x) f_{i}\left(u_{i}(x)\right)=0, i=1,2, \quad x \in \Omega_{i} \\
D_{1} \frac{\mathrm{d} u_{1}(0)}{\mathrm{d} x}=D_{2} \frac{\mathrm{d} u_{2}(0)}{\mathrm{d} x} \\
u_{1}(0)=k u_{2}(0) \\
\frac{\mathrm{d} u_{1}\left(-L_{1}\right)}{\mathrm{d} x}=\frac{\mathrm{d} u_{2}\left(L_{2}\right)}{\mathrm{d} x}=0
\end{array}\right.
$$

In the next section, we prove the uniqueness, existence, and global stability of the steady state.

\section{Existence and global stability of a positive steady state}

In this section, we first rescale our model and use some ideas by Freedman et al. (1989) to prove existence and uniqueness of a positive solution of the steady-state problem (10). Then we prove that this positive solution is globally asymptotically stable, using monotonicity properties of the system. Finally, we classify the possible qualitative shapes of the positive steady-state solutions.

\subsection{Scaling and continuous solutions}

Freedman et al. (1989) considered a steady-state problem of population dynamics on two adjacent patches, similar to our problem. Their model differed from ours in that they assumed continuity at the interface and a different set of boundary conditions. In order to apply their ideas, we first scale our model to obtain continuous interface conditions.

Following Maciel and Lutscher (2015), we define the new variables for the time-dependent problem

$$
\left\{\begin{array}{l}
v_{1}(\xi, t)=u_{1}(x, t), \quad \xi=x \in \widetilde{\Omega}_{1}:=\left[-L_{1}, 0\right] \\
v_{2}(\xi, t)=k u_{2}(x, t), \quad \xi=\frac{x}{k} \in \widetilde{\Omega}_{2}:=\left[0, \frac{L_{2}}{k}\right] .
\end{array}\right.
$$


In this new scaling, system (9) takes the form

$$
\begin{cases}\frac{\partial v_{1}(\xi, t)}{\partial t}=\widetilde{D}_{1} \frac{\partial^{2} v_{1}(\xi, t)}{\partial \xi^{2}}+v_{1}(\xi, t) \widetilde{f}_{1}\left(v_{1}(\xi, t)\right), & \text { on } \widetilde{\Omega}_{1} \times[0, \infty) ; \\ \frac{\partial v_{2}(\xi, t)}{\partial t}=\widetilde{D}_{2} \frac{\partial^{2} v_{2}(\xi, t)}{\partial \xi^{2}}+v_{2}(\xi, t) \widetilde{f}_{2}\left(v_{2}(\xi, t)\right), & \text { on } \widetilde{\Omega}_{2} \times[0, \infty) ; \\ \widetilde{D}_{1} \frac{\partial v_{1}(0, t)}{\partial \xi}=\widetilde{D}_{2} \frac{\partial v_{2}(0, t)}{\partial \xi}, & t \geq 0 ; \\ \frac{\partial v_{1}\left(-L_{1}, t\right)}{\partial \xi}=\frac{\partial v_{2}\left(L_{2} / k, t\right)}{\partial \xi}=0, & t \geq 0 ; \\ v_{1}(0, t)=v_{2}(0, t), & t \geq 0 ;\end{cases}
$$

where $\widetilde{D}_{1}=D_{1}, \widetilde{D}_{2}=\frac{D_{2}}{k^{2}}, \widetilde{f}_{1}\left(v_{1}\right)=f_{1}\left(v_{1}\right)$, and $\widetilde{f}_{2}\left(v_{2}\right)=f_{2}\left(v_{2} / k\right)$. We note that in this scaling, we have continuity of density and flux at the interface.

We denote $\tilde{L}_{2}=\frac{L_{2}}{k}, \widetilde{K}_{1}=K_{1}$ and $\widetilde{K}_{2}=k K_{2}$. For simplicity of notation, we drop the $\sim$ from here on and revert to the original variable name $\xi=x$. As a result, we rewrite the above system as

$$
\begin{cases}\frac{\partial v_{1}(x, t)}{\partial t}=D_{1} \frac{\partial^{2} v_{1}(x, t)}{\partial x^{2}}+v_{1}(x, t) f_{1}\left(v_{1}(x, t)\right), & \text { on } \Omega_{1} \times[0, \infty) \\ \frac{\partial v_{2}(x, t)}{\partial t}=D_{2} \frac{\partial^{2} v_{2}(x, t)}{\partial x^{2}}+v_{2}(x, t) f_{2}\left(v_{2}(x, t)\right), & \text { on } \Omega_{2} \times[0, \infty) \\ D_{1} \frac{\partial v_{1}(0, t)}{\partial x}=D_{2} \frac{\partial v_{2}(0, t)}{\partial x}, & t \geq 0 ; \\ \frac{\partial v_{1}\left(-L_{1}, t\right)}{\partial x}=\frac{\partial v_{2}\left(L_{2}, t\right)}{\partial x}=0, & t \geq 0 ; \\ v_{1}(0, t)=v_{2}(0, t), & t \geq 0 .\end{cases}
$$


3.2 Existence and uniqueness of the positive steady state

In the rescaled variables, system (10) assumes the form:

$$
\begin{cases}D_{1} \frac{\mathrm{d}^{2} v_{1}(x)}{\mathrm{d} x^{2}}+v_{1}(x) f_{1}\left(v_{1}(x)\right)=0, & \text { on } \Omega_{1}=\left[-L_{1}, 0\right] \\ D_{2} \frac{\mathrm{d}^{2} v_{2}(x)}{\mathrm{d} x^{2}}+v_{2}(x) f_{2}\left(v_{2}(x)\right)=0, & \text { on } \Omega_{2}=\left[0, L_{2}\right] \\ D_{1} \frac{\mathrm{d} v_{1}(0)}{\mathrm{d} x}=D_{2} \frac{\mathrm{d} v_{2}(0)}{\mathrm{d} x}, v_{1}(0)=v_{2}(0) ; & \\ \frac{\mathrm{d} v_{1}\left(-L_{1}\right)}{\mathrm{d} x}=0, \frac{\mathrm{d} v_{2}\left(L_{2}\right)}{\mathrm{d} x}=0\end{cases}
$$

which is, of course, the steady-state problem of the rescaled dynamic system (13).

We note that if $K_{2}=K_{1}$, the function $v_{1}(x)=v_{2}(x)=K_{1}$ is a solution of (14). The challenge is to find a solution when $K_{1} \neq K_{2}$. Without loss of generality, we assume that $K_{2}>K_{1}$. Our main result in this section is the following.

Theorem 1 There exists a unique positive solution of system (14), and this solution is monotone on each $\Omega_{i}$.

We begin the proof of this theorem with some general considerations. If we suppose that $0<v_{1}\left(-L_{1}\right) \leq K_{1}<K_{2}$ then, by the maximum principle, we necessarily have $v_{1}^{\prime}(0) \leq 0$. By continuity, we then find $v_{2}(0)<K_{2}$ and $v_{2}^{\prime}(0) \leq 0$. By the maximum principle again, this implies that $v_{2}^{\prime}\left(L_{2}\right)<0$ so that the boundary conditions cannot be met. Hence, we must have $v_{1}\left(-L_{1}\right)>K_{1}$. The same reasoning applies to show that necessarily $v_{2}\left(L_{2}\right)<K_{2}$. We now construct our desired solution such that $K_{1}<v_{1}\left(-L_{1}\right)<v_{2}\left(L_{2}\right)<K_{2}$. We split the construction into several smaller steps that follow the ideas of Freedman et al. (1989). We only give the proofs for those statements that are not found in their paper.

We define $q_{1}\left(x, \alpha_{1}\right), x \in \Omega_{1}$ to be the unique solution of the first equation of (14) such that

$$
\frac{\partial q_{1}\left(-L_{1}, \alpha_{1}\right)}{\partial x}=0, \quad q_{1}\left(-L_{1}, \alpha_{1}\right)=\alpha_{1},
$$

and $q_{2}\left(x, \alpha_{2}\right), x \in \Omega_{2}$ the unique solution of the second equation of (14) such that

$$
\frac{\partial q_{2}\left(L_{2}, \alpha_{2}\right)}{\partial x}=0, \quad q_{2}\left(L_{2}, \alpha_{2}\right)=\alpha_{2}
$$

Existence and uniqueness of $q_{i}$ is guaranteed by the general existence and uniqueness theorem for ordinary differential equations; see e.g. Perko (2001).

If we can show that there exist unique $\alpha_{1}, \alpha_{2}$ such that

$$
\left\{\begin{array}{c}
q_{1}\left(0, \alpha_{1}\right)=q_{2}\left(0, \alpha_{2}\right), \\
D_{1} \frac{\partial q_{1}\left(0, \alpha_{1}\right)}{\partial x}=D_{2} \frac{\partial q_{2}\left(0, \alpha_{2}\right)}{\partial x}
\end{array}\right.
$$

then we have shown the existence and uniqueness of a positive solution of model (14). The following three lemmas are key to achieve this goal. 
Lemma 1 If $\alpha_{1}>K_{1}$ then

$$
\frac{\partial q_{1}\left(x, \alpha_{1}\right)}{\partial x}>0 \quad \text { on } \quad-L_{1}<x \leqslant 0 .
$$

Lemma 2 If $\alpha_{2}<K_{2}$ then

$$
\frac{\partial q_{2}\left(x, \alpha_{2}\right)}{\partial x}>0 \quad \text { on } \quad 0 \leqslant x<L_{2} .
$$

Lemma 3 Define $H_{i}\left(\alpha_{i}\right):=q_{i}\left(0, \alpha_{i}\right), i=1,2$. Then there exist $\hat{\alpha}_{i}$ such that

$$
H_{1}:\left[K_{1}, \hat{\alpha}_{1}\right] \rightarrow\left[K_{1}, K_{2}\right] \quad \text { and } \quad H_{2}:\left[\hat{\alpha}_{2}, K_{2}\right] \rightarrow\left[K_{1}, K_{2}\right]
$$

Furthermore, $H_{i}$ are monotone functions. In particular, for every $\alpha_{1} \in\left[K_{1}, \hat{\alpha}_{1}\right]$ there exists a unique $\alpha_{2} \in\left[\hat{\alpha}_{2}, K_{2}\right]$, such that $q_{1}\left(0, \alpha_{1}\right)=q_{2}\left(0, \alpha_{2}\right)$. In particular, there exists a continuous solution.

These lemmas ensure the existence of solutions that are continuous. To ensure the existence of a solution that also satisfies the flux condition at $x=0$, Freedman et al. (1989) apply the intermediate value theorem. Their proof carries over to our case as well. This completes the proof of Theorem 1 .

It remains to prove the lemmas. The proof of Lemma 3 is identical to the one in Freedman et al. (1989), but the other two differ somewhat. We only provide the proof of Lemma 2 here, the proof of Lemma 1 is essentially the same.

Proof : Based on (16) and the assumption of this lemma, we have $q_{2}\left(L_{2}, \alpha_{2}\right)=$ $\alpha_{2}<K_{2}$. By continuity, we can assume that there exists a small enough value $\varepsilon>0$ such that $q_{2}\left(x, \alpha_{2}\right)<K_{2}$ on the interval $\left(L_{2}-\varepsilon, L_{2}\right]$. From the second equation of system (14), we see that $\frac{\partial^{2} q_{2}\left(x, \alpha_{2}\right)}{\partial x^{2}}<0$ so that we conclude $\frac{\partial q_{2}\left(x, \alpha_{2}\right)}{\partial x}>0$ on this interval. On the other hand, by (16) we have $\frac{\partial q_{2}\left(L_{2}, \alpha_{2}\right)}{\partial x}=0$.

Thus, $\frac{\partial q_{2}\left(x, \alpha_{2}\right)}{\partial x}>0$, which shows that $q_{2}\left(x, \alpha_{2}\right)$ is an increasing function on the interval $\left(L_{2}-\varepsilon, L_{2}\right]$. If $q_{2}\left(x, \alpha_{2}\right)$ is not increasing everywhere in $\left[0, L_{2}\right]$, we can conclude that there exists a point, $\bar{x}$, such that $\frac{\partial q_{2}\left(\bar{x}, \alpha_{2}\right)}{\partial x}=0$. By construction, $q_{2}$ satisfies the second equation in (14). We multiply this equation by $\mathrm{d} q_{2} / \mathrm{d} x$ and integrate from 0 to $L_{2}$. We obtain

$$
\left(\frac{\partial q\left(x, \alpha_{2}\right)}{\partial x}\right)^{2}=-\frac{2}{D_{2}} \int_{\alpha_{2}}^{q_{2}\left(\bar{x}, \alpha_{2}\right)} \zeta f_{2}(\zeta) d \zeta .
$$

If we evaluate this expression at $\bar{x}$, then the left-hand side is zero while the righthand side is negative since $\zeta>\alpha_{2}$. Thus, we get a contradiction.

Freedman et al. (1989) proved that the steady state of (14) is locally asymptotically stable under the sufficient condition that $\frac{d}{d v}(v f(v))<0$ for $K_{1} \leq v \leq K_{2}$. It will become clear below that this condition is much too strong for many of the cases that we are interested in. Therefore, we prove the much stronger result that the positive steady state is unconditionally stable. 
Theorem 2 The positive steady-state solution of (13) is globally asymptotically stable.

Proof The proof is a slight adaptation of the proof of Proposition 3.2 in Cantrell and Cosner (2003). Maciel et al. (2019) showed the existence and uniqueness of solutions of (13), using semigroup theory. They also showed that the equation possesses a comparison principle. Furthermore, they showed that the linearization of (13) at a steady state possesses a dominant eigenvalue with positive eigenfunction. We find that $\max \left\{K_{1}, K_{2}\right\}$ is a supersolution for our model. As in Proposition 3.2 in Cantrell and Cosner (2003), we can construct a subsolution as an appropriate multiple of the eigenfunction of the linearization at zero. By the calculations in Maciel and Lutscher (2013), this eigenvalue is positive for the chosen functions $f_{i}$. Hence, the trivial steady state is unstable, and the subsolution will converge upward to a smallest positive steady state. Since the steady state is unique, our claim follows.

In the following section, we illustrate how the qualitative behavior of shapes of the positive steady state for system (10) depends on parameters.

3.3 Classifying the shapes of the positive steady state

We illustrate how the value of the composite parameter (see (8))

$$
k=\frac{p_{1}}{1-p_{1}} \frac{D_{2}}{D_{1}}
$$

affects the steady state of the system. We choose patch preference $\left(p_{1}\right)$ as our parameter of interest here. We discuss the influence of the diffusion rates in a different context below.

For reference, we summarize the results from Section 3.2. To avoid confusion, we will use the notation from (12) with tildes. According to Theorem 1, there are three possible shapes of the solution.

a) If $\widetilde{K}_{1}=\widetilde{K}_{2}$, then the solution is constant in space.

b) If $\widetilde{K}_{1}<\widetilde{K}_{2}$ then the solution is increasing with $v_{1}>\widetilde{K}_{1}$, and $v_{2}<\widetilde{K}_{2}$.

c) If $\widetilde{K}_{1}>\widetilde{K}_{2}$ then the solution is decreasing with $v_{1}<\widetilde{K}_{1}$, and $v_{2}>\widetilde{K}_{2}$.

A direct translation of these properties to the unscaled steady-state problem (10) gives the following possible shapes.

Lemma 4 The positive solution of (10) has exactly one of the following shapes.

$\left.a^{\prime}\right)$ If $K_{1}=k K_{2}$ then the solution is constant on each patch.

$\left.b^{\prime}\right)$ If $K_{1}<k K_{2}$ then the solution is increasing on each patch with $u_{1}>K_{1}$ and $u_{2}<K_{2}$.

$\left.c^{\prime}\right)$ If $K_{1}>k K_{2}$ then the solution is decreasing on each patch with $u_{1}<K_{1}$ and $u_{2}>K_{2}$.

Solutions of the unscaled model are not continuous at the interface, unless $k=$ 1. Even if the solution is monotone on each patch, the jump at the interface may be in the "opposite" direction so that the solution may not be monotone over the 
entire landscape. We illustrate how the value of $k$ affects the steady-state solution in general and the jump at the interface in particular. We fix all parameters, except for $p_{1}$ and we choose $K_{2}>K_{1}$, without loss of generality. As we decrease $p_{1}, k$ also decreases. According to the three cases above, we find three cases for the value of $k$, see Figure 1 . When $k>1$, the solution is monotone increasing in each patch but jumps down at the interface; see Figure 2(a). As $k$ decreases, the jump at the interface decreases until the solution becomes continuous at $k=1$ (plot not shown). As long as $k>K_{1} / K_{2}$, the solution remains increasing in each patch. Since the jump is now up, the solution is monotone on the entire landscape; see Figure 2(b). When $k=K_{1} / K_{2}$, the solution is piecewise constant, equal to the carrying capacity in each patch (plot no shown). When $k<K_{1} / K_{2}$ the jump at the interface is larger than the jump in the carrying capacities. In this case, the solution is decreasing on each patch. Since the jump at the interface is up, the solution is not globally decreasing. In fact, the solution now exceeds the larger of the two carrying capacities; see Figure 2(c). Finally, we briefly illustrate the case where $K_{1}=K_{2}$. In this case, we have $k>1$ exactly when $k>K_{1} / K_{2}$, so that the three cases in Figure 1 collapse to only two. When $k>1$, the solution is increasing in each patch and the jump is down, so that the solution is not globally monotone. Necessarily, the solution exceeds the maximum of the carrying capacity; see Figure $2(d)$.

$$
\mathrm{k} \cdot \mathrm{png}
$$

Fig. 1 Three different cases for the parameter of discontinuity at the interface, $k$, where $K_{2}>K_{1}$

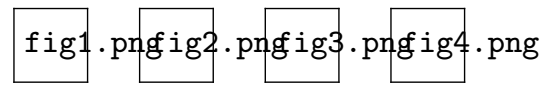

$(\mathrm{a})$
$p_{1}=\begin{aligned} & (\mathrm{b}) \\ & p_{1} \\ & 0.6\end{aligned}=\begin{aligned} & (\mathrm{c}) \\ & p_{1} \\ & 0.2\end{aligned}=\begin{aligned} & (\mathrm{d}) \\ & K_{1}= \\ & K_{2} \text { and } \\ & p_{1}= \\ & 0.6\end{aligned}=$

Fig. 2 We illustrate how the shape of the steady-state solution of system (10) changes as the patch preference $\left(p_{1}\right)$ changes. Top left: When $p_{1}=0.6$, we have Case 1 with $k=2.25>1$. Top right: When $p_{1}=0.35$, we have Case 2 with $K_{1} / K_{2}=0.625<0.8077=k<1$. Bottom left: When $p_{1}=0.2$, we have Case 3 with $k=0.3750<K_{1} / K_{2}$. Bottom right: When $K_{1}=K_{2}$, the two threshold conditions for $k$ are identical. When $p_{1}=0.6$, we are in Case 1 with $k=2.25$. See text for detailed description. We chose the patches $\Omega_{1}=[-6,0], \Omega_{2}=[0,6]$. Parameters are $K_{1}=5=r_{1}, K_{2}=8=r_{2}, D_{1}=2$ and $D_{2}=3$ unless otherwise indicated. The density in patch 1 (patch 2) is plotted in blue (red). The dashed lines correspond to the respective carrying capacities.

From a biological point of view, we see that when individuals have a high preference for patch 1, they will pour into patch one and thereby increase the steady-state density in patch 1 near the interface above its carrying capacity. In 
fact, the density on the entire patch will then be above its carrying capacity. At the same time, individuals leave patch 2 so that the density in that patch is decreased at the interface and therefore also in the entire patch. When the preference for patch 1 is low, the situation is reversed.

\section{Role of movement in steady-state density}

The illustrations in Figure 2 show that the steady-state density is greater than the local carrying capacity in one of the two patches and less than the local carrying capacity in the other patch. We are interested in whether the gain in population density over the carrying capacity in one patch exceeds the loss on the other patch or not. The magnitude of this effect depends on individual movement behavior at the interface through the composite parameter $k$ in (8). Hence, we are led to ask whether individual movement behavior benefits the population at steady state and what the underlying mechanisms are. It is clear that in the absence of any movement, the steady-state density equals carrying capacity everywhere.

As mentioned in the introduction, this question has been studied in the context of population dynamics in discrete habitat patches (Freedman and Waltman, 1977). The first such study in the context of reaction-diffusion equations that we are aware of is by Lou (2006). Lou studied the steady-state density of (1) under the assumption that $r(x)=K(x)$ is not constant. He showed that inequality (2) holds for all $D>0$. DeAngelis et al. (2016) studied the same question in the case where $r(x) \neq K(x)$ is not constant. They found that inequality (2) holds under the additional assumption that $r$ and $K$ are positively correlated in space, at least when $D$ is small. In both works, the authors assumed that $D>0$ is independent of local conditions.

It turns out that the basic result by Lou (2006) is still true when the diffusion term in (1) is replaced by spatially varying diffusion of Fickian type.

Lemma 5 Consider the positive solution of

$$
\frac{\mathrm{d}}{\mathrm{d} x}\left(D(x) \frac{\mathrm{d} u}{\mathrm{~d} x}\right)+u(K(x)-u)=0
$$

on $[0, L]$ with positive and sufficiently smooth functions $D(\cdot)$ and $K(\cdot)$ and no-flux boundary conditions

$$
D(0) \frac{\mathrm{d} u}{\mathrm{~d} x}(0)=0, \quad D(L) \frac{\mathrm{d} u}{\mathrm{~d} x}(L)=0
$$

Then

$$
\int_{0}^{L}[u(x)-K(x)] d x>0 .
$$

The proof of this lemma is only a slight generalization of the proof in Lou (2006) and is given in the appendix for completeness. In the same appendix, we also show that the statement is generally not true when we replace the Fickian diffusion term with an ecological diffusion term (Turchin, 1998). The calculations in the appendix also show that there is no simple condition under which inequality (2) would hold in the latter case. 
The assumption that movement behavior is independent of local conditions is highly unrealistic in natural systems. Most organisms adjust their movement behavior to the quality of the habitat that they are in, and often also direct their movement to certain types of habitat. For a recent review of some empirical evidence, see Crone et al. (2019). In models with continuously varying landscape quality, such as (18), determining the form of $D(x)$ and/or estimating it from data is a complicated endeavour. Our modelling approach with piecewiece constant functions and a single parameter at an interface offers a much more manageable alternative. In fact, empirical estimates of habitat preferences are quite common in the literature, see e.g. references in Maciel and Lutscher (2013).

In our model, we calculated the difference between the total steady-state density and the total carrying capacity for the four plots in Figure 2; see Table 1. We find that inequality (2) holds only in one of the four cases. We will derive a sufficient condition for the inequality to hold.

\begin{tabular}{cccc}
\hline \hline Fig 2 & $\int_{\Omega} u(x) \mathrm{d} x$ & $\int_{\Omega} K(x) \mathrm{d} x$ & $\int_{\Omega}[u(x)-K(x)] \mathrm{d} x$ \\
\hline \hline (a) & 77.686 & 78 & -0.31417 \\
(b) & 78.168 & 78 & 0.16754 \\
(c) & 77.442 & 78 & -0.55800 \\
(d) & 59.505 & 60 & -0.49502 \\
\hline
\end{tabular}

Table 1 Numerical evaluation of the total population density at steady state, the total carrying capacity, and their difference for the four plots in Figure 2. Only in case (b) inequality (2) is satisfied.

\subsection{Total density at steady state}

To derive a sufficient condition for inequality (2) to hold in our system, we consider the positive solution of our steady-state equations as usual

$$
\left\{\begin{array}{l}
D_{1} \frac{\mathrm{d}^{2} u_{1}(x)}{\mathrm{d} x^{2}}+u_{1} f_{1}\left(u_{1}\right)=0, \quad x \in \Omega_{1}=\left[-L_{1}, 0\right] \\
D_{2} \frac{\mathrm{d}^{2} u_{2}(x)}{\mathrm{d} x^{2}}+u_{2} f_{2}\left(u_{2}\right)=0, \quad x \in \Omega_{2}=\left[0, L_{2}\right] \\
D_{1} \frac{\mathrm{d} u_{1}(0)}{\mathrm{d} x}=D_{2} \frac{\mathrm{d} u_{2}(0)}{\mathrm{d} x}, u_{1}(0)=k u_{2}(0) \\
\frac{\mathrm{d} u_{1}\left(-L_{1}\right)}{\mathrm{d} x}=0, \frac{\mathrm{d} u_{2}\left(L_{2}\right)}{\mathrm{d} x}=0 .
\end{array}\right.
$$

We divide the first equation by $u_{1}$ and integrate. We find

$$
\frac{D_{1}}{u_{1}(0)} \frac{\mathrm{d} u_{1}(0)}{\mathrm{d} x}+D_{1} \int_{-L_{1}}^{0} \frac{1}{u_{1}^{2}(x)} \frac{\mathrm{d} u_{1}(x)^{2}}{\mathrm{~d} x} \mathrm{~d} x+\int_{-L_{1}}^{0} f_{1}\left(u_{1}(x)\right) \mathrm{d} x=0
$$


Similarly, dividing the second equation in (19) by $u_{2}$ and integrating gives

$$
-\frac{D_{2}}{u_{2}(0)} \frac{\mathrm{d} u_{2}(0)}{\mathrm{d} x}+D_{2} \int_{0}^{L_{2}} \frac{1}{u_{2}^{2}(x)} \frac{\mathrm{d} u_{2}(x)^{2}}{\mathrm{~d} x} \mathrm{~d} x+\int_{0}^{L_{2}} f_{2}\left(u_{2}(x)\right) \mathrm{d} x=0 .
$$

Now we use $f_{i}(u)=r_{i}\left(1-u / K_{i}\right)=r_{i}\left(K_{i}-u\right) / K_{i}$. We multiply $(20)$ by $K_{1} / r_{1}$ and (21) by $K_{2} / r_{2}$ and add the two. We obtain

$$
\int_{-L_{1}}^{0}\left[u_{1}(x)-K_{1}\right] \mathrm{d} x+\int_{0}^{L_{2}}\left[u_{2}(x)-K_{2}\right] \mathrm{d} x=I_{1}+I_{2}
$$

where

$$
I_{1}=\frac{K_{1} D_{1}}{r_{1}} \int_{-L_{1}}^{0} \frac{1}{u_{1}^{2}(x)} \frac{\mathrm{d} u_{1}(x)^{2}}{\mathrm{~d} x} \mathrm{~d} x+\frac{K_{2} D_{2}}{r_{2}} \int_{0}^{L_{2}} \frac{1}{u_{2}^{2}(x)} \frac{\mathrm{d} u_{2}(x)^{2}}{\mathrm{~d} x} \mathrm{~d} x
$$

and

$$
I_{2}=\frac{K_{1}}{r_{1}} \frac{D_{1}}{u_{1}(0)} \frac{\mathrm{d} u_{1}(0)}{\mathrm{d} x}-\frac{K_{2}}{r_{2}} \frac{D_{2}}{u_{2}(0)} \frac{\mathrm{d} u_{2}(0)}{\mathrm{d} x} .
$$

The left-hand side in (22) is the quantity that we are interested in: the difference between the total density and the total carrying capacity. The first term on the right-hand side is clearly positive. Using the interface conditions, we simplify the second term into

$$
I_{2}=\frac{D_{1}}{u_{1}(0)} \frac{\mathrm{d} u_{1}(0)}{\mathrm{d} x}\left(\frac{K_{1}}{r_{1}}-k \frac{K_{2}}{r_{2}}\right),
$$

with $k$ as in (8). Hence, we have the following result.

Theorem 3 Let $u_{1}, u_{2}$ be the positive solution of (19) with logistic growth terms $f_{i}(u)=r_{i}\left(1-u / K_{i}\right)$. If

$$
\frac{\mathrm{d} u_{1}(0)}{\mathrm{d} x}\left(1-\frac{p_{1}}{1-p_{1}} \frac{D_{2}}{D_{1}} \frac{K_{2}}{r_{2}} \frac{r_{1}}{K_{1}}\right)=\frac{\mathrm{d} u_{1}(0)}{\mathrm{d} x}\left(1-k \frac{K_{2}}{K_{1}} \frac{r_{1}}{r_{2}}\right)>0,
$$

then

$$
\int_{-L_{1}}^{0}\left[u_{1}(x)-K_{1}\right] \mathrm{d} x+\int_{0}^{L_{2}}\left[u_{2}(x)-K_{2}\right] \mathrm{d} x>0,
$$

i.e., the total population density is higher than the total carrying capacity.

The condition in the theorem simplifies significantly if we assume $r_{i}=K_{i}$, as was done by Lou (2006), or, slightly more generally, if $K_{2} / K_{1}=r_{2} / r_{1}$.

Corollary 1 Assume that $K_{2} / K_{1}=r_{2} / r_{1}$. If $K_{2}>K_{1}$, then inequality (27) holds if $K_{1} / K_{2}<k<1$. Similarly, if $K_{1}>K_{2}$ then inequality (27) holds if $K_{1} / K_{2}>k>1$.

Proof When $K_{2} / K_{1}=r_{2} / r_{1}$, condition (26) holds if and only if $\mathrm{d} u_{1}(0) / \mathrm{d} x$ and $(1-k)$ have the same sign. Assume $K_{2}>K_{1}$. According to Lemma 4, the solution is increasing on each patch if $k>K_{1} / K_{2}$. Hence, $\mathrm{d} u_{1}(0) / \mathrm{d} x>0$. If also $k<1$ then both factors of (26) are positive. The other case is similar. 
To illustrate this result, we return to Figure 2. Parameters were chosen such that $r_{i}=K_{i}$ and $K_{2}>K_{1}$. Hence, only plot (b) satisfies the conditions of the preceding corollary. We see from Table 1 that the integral inequality does indeed hold for that case, but not for any other.

In the general case, Lemma 4 gives us the sign of $\mathrm{d} u_{1} / \mathrm{d} x>0$ as a function of $K_{1}, K_{2}$ and $k$. Combined with Theorem 3, we find that inequality (27) holds in the following two cases

$$
1<k \frac{K_{2}}{K_{1}}<\frac{r_{2}}{r_{1}} \quad \text { and } \quad 1>k \frac{K_{2}}{K_{1}}>\frac{r_{2}}{r_{1}}
$$

To show that these conditions are sufficient but not necessary, we present another set of numerical results, this time with $r_{i} \neq K_{i}$. Instead of varying parameter $p_{1}$ (the probability that an individual at the interface chooses to move into patch 1 ), we vary the diffusion coefficients in the two patches. The set-up is the same as in Figure 2, only the parameter values are different (see figure caption). The conditions from Theorem 3 are satisfied only in panel (b), yet inequality (27) is satisfied for (b) and (d) as Table 2 shows. In panels (a) and (c), the value of $k K_{2} / K_{1}$ is an order of magnitude smaller or larger, respectively, than unity. In those cases, inequality (27) cannot be satisfied.

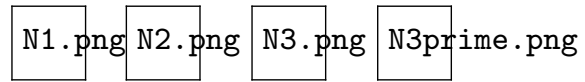

Fig. 3 We illustrate how the shape of the steady-state solution of system (10) changes as diffusion coefficients change. Top left: We have $k K_{2} / K_{1}=0.16<1<1.5=r_{2} / r_{1}$. Top right: We have $1<k K_{2} / K_{1}=1.2<1.5=r_{2} / r_{1}$. Bottom left: We have $k K_{2} / K_{1}=24>1.5=$ $r_{2} / r_{1}>1$. Bottom right: We have $k K_{2} / K_{1}=2>1.5=r_{2} / r_{1}>1$. Common parameters are $r_{1}=4, r_{2}=6$, and $p_{1}=0.5$. Other parameters are as in Figure 2 .

\begin{tabular}{cccc}
\hline \hline Fig 3 & $\int_{\Omega} u(x) \mathrm{d} x$ & $\int_{\Omega} K(x) \mathrm{d} x$ & $\int_{\Omega}(u(x)-K(x)) \mathrm{d} x$ \\
\hline \hline (a) & 73.531 & 78 & -4.4691 \\
(b) & 78.148 & 78 & 0.14769 \\
(c) & 70.292 & 78 & -7.7085 \\
(d) & 78.136 & 78 & 0.13557 \\
\hline
\end{tabular}

Table 2 Numerical evaluation of the total population density at steady state, the total carrying capacity, and their difference for the four plots in Figure 3. Inequality (2) is satisfied in cases (b) and (d) even though the sufficient conditions from Theorem 3 are satisfied only for (b).

To further clarify the conditions under which inequality (27) can hold, we plot the difference between the total steady-state density and the total carrying 
capacity as a function of parameter $k$; see Figure 4 . We see that the inequality is satisfied for some intermediate range of $k$, but not for very small or very large $k$. We also see that the range in which the inequality holds (where the graph is positive) is larger than what the theorem predicts (between the two dashed vertical lines). In particular, while the lower bound, $k>K_{1} / K_{2}$, seems to be optimal, the upper bound, $k<K_{1} r_{2} /\left(K_{2} r_{1}\right)$, is clearly not optimal for the chosen parameters. We return to this question later. When $k<K_{1} / K_{2}$, the steady state exceeds the higher of the two carrying capacities and is smaller than the lower of the two near the interface. In this case, the total density is always below the total carrying capacity. On the other hand, if movement behavior is such that the density exceeds the lower carrying capacity and remains below the higher of the two, then the total effect is positive for the population density, at least as long as the effect is not too large.

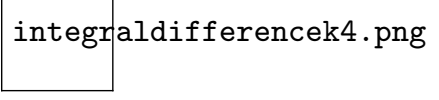

Fig. 4 Plot of the integral difference $\int_{\Omega}(u(x)-K(x)) \mathrm{d} x$ with respect to $\log (k)$. The black and red dashed lines indicate the sufficient conditions from the theorem, $\frac{K_{1}}{K_{2}}<k<\frac{K_{1} r_{2}}{K_{2} r_{1}}$.

We can relate a special case of our results to those by DeAngelis et al. (2016). If we assume, as those authors did, that diffusion is constant throughout the two patches and that there is no preference for any of the patches, then we have $k=1$. If we further assume (without loss of generality) that $K_{2}>K_{1}$, then for inequality (27) to hold, we require $r_{2} / r_{1}>K_{2} / K_{1}>1$. In particular, $r_{i}$ and $K_{i}$ have to be positively correlated, as was the case for DeAngelis et al. (2016). However, while those authors obtained their result only for a small enough diffusion coefficient, our reasoning applies without that restriction, but for a much simpler landscape than theirs.

We conclude this section with another observation in Figure 3. When the two diffusion coefficients differ by an order of magnitude, there is a clearly visible difference in the maximum slope and curvature of the steady-state solution. For example, whenever $D_{i}$ is small, the solution is close to the carrying capacity (and hence nearly constant) for much of the patch (e.g., the red curve in panel (a) and the blue curve in panel (c)). Whenever $D_{i}$ is large, the maximum curvature is smaller, and the solution changes more gradually (e.g., the blue curve in panel (a) and the red curve in panel (c)). In the next section, we study the shape of the steady-state density in the extreme cases where both diffusion coefficients become very small or both become very large.

\subsection{The limits of fast and slow diffusion}

So far, we only examined one aspect of the results by Lou (2006) in our two-patch model, namely his result that if $r(x)=K(x)$ in (1) then inequality (2) holds. We already found significant differences in our model that relate to individual movement behavior. But Lou's results are much finer than inequality (2). He proves 


\section{S2prime.png}

Fig. 5 Steady-state profiles of the two-patch model for varying values of $D_{1}$ with constant ratio $D_{2} / D_{1}=0.9$. As $D_{1}$ approaches zero, the solution is close to the carrying capacity on each patch (indicated by the black dashed lines) and has a narrow but sharp transition zone near the interface (the lowest of the blue curves and the highest of the red curves correspond to the smallest value $D_{1}=0.1$ ). As $D_{1}$ gets large, the steady-state solution becomes increasingly flat and approaches the constant values $u_{i}^{*}$ from (28) on patch $i$ (indicated by the black solid lines). Other parameters are as in Figure 3.

that as $D \rightarrow 0$, the steady-state density approaches the carrying capacity in the $L^{p}$-norm for all $p \geq 1$. He also proves that as $D \rightarrow \infty$, the steady-state density approaches a constant, given by the spatial average of the carrying capacity, in the $W^{2, p}$-norm for $p \geq 1$. In this final section, we formulate analogous questions for our two-patch model and give numerical and heuristic evidence that analogous results could hold.

The first question is how to formulate the problem since model (1) contains only one diffusion coefficient, but our two-patch model (3) contains two. We choose to vary $D_{1,2}$ in such a way that their ratio, $d=D_{1} / D_{2}$, remains constant. Then parameter $k$ remains independent of $D_{i}$.

We present some numerical experiments. The plot in Figure 5 show that as $D_{i} \rightarrow 0$, the steady-state density approaches the carrying capacity on each patch with an increasingly steep transition zone near the interface. As $D_{i} \rightarrow \infty$, we see that the steady-state density approaches a constant on each patch. In contrast to the result by Lou (2006), this constant is generally not the same throughout the entire domain, because the matching conditions at the interface need to be satisfied.

We can derive a heuristic expression for the densities in each patch in the limit as $D_{i} \rightarrow \infty$. We denote by $u_{i}^{*}$ the spatially constant steady-state density on patch $i$. Then we integrate the equations over both patches and find

$$
L_{1} r_{1} u_{1}^{*}\left(1-\frac{u_{1}^{*}}{K_{1}}\right)+L_{2} r_{2} u_{2}^{*}\left(1-\frac{u_{2}^{*}}{K_{2}}\right)=0 .
$$

Substituting the interface condition $u_{1}^{*}=k u_{2}^{*}$ gives the solution

$$
u_{2}^{*}=\frac{L_{1} r_{1} k+L_{2} r_{2}}{\frac{L_{1} r_{1} k}{K_{1} / k}+\frac{L_{2} r_{2}}{K_{2}}}, \quad u_{1}^{*}=k u_{2}^{*} .
$$

Upon closer inspection, we see that (28) is the harmonic mean of $K_{2}$ and $K_{1} / k$ with weights $L_{2} r_{2}$ and $L_{1} r_{1} k$. This result is closely related to the work by Yurk and Cobbold (2018). Those authors considered population dynamics in an infinite landscape of periodically alternating patches of two types. They derived equations for the homogenization limit when the patch sizes $L_{1,2} \rightarrow 0$. In that limit, the carrying capacity turned out the be an appropriately weighted harmonic average of the patch-level carrying capacities. A steady-state solution of our model on just two patches is equivalent to a periodic solution of their model with twice the patch size because of the no-flux conditions at $-L_{1}$ and $L_{2}$; see also Maciel et al. (2019) for a similar argument. Our assumption that $D_{i} \rightarrow \infty$ can be turned into the homogenization assumption $L_{i} \rightarrow 0$ by a simple rescaling of space. 
For the solution in (28), we can explicitly calculate exact conditions for the total density to exceed the total carrying capacity. We have

$$
L_{1} u_{1}^{*}+L_{2} u_{2}^{*}>L_{1} K_{1}+L_{2} K_{2}
$$

if and only if

$$
\frac{K_{1}}{K_{2}}<k<\frac{K_{1}}{K_{2}} \frac{r_{2}}{r_{1}}
$$

In other words, in the limit of fast diffusion, the upper bound that we found from Theorem 3 for inequality (26) to hold is sharp.

We illustrate three possible behaviors of the difference between the total steadystate density and the total carrying capacity as a function of diffusion rates in Figure 6. When $k<K_{1} / K_{2}$, the total steady-state density is below the total carrying capacity for all values of $D_{1}$ (dashed curve). The curve is monotone decreasing and approaches the value given by (28). When (29) is satisfied, the total steady-state density is above the total carrying capacity for all $D_{1}$ (solid curve). The curve has an intermediate maximum as in the case studied by Lou (2006). In the limit, however, we obtain again the values from (28), whereas Lou (2006) found that the total steady-state density equals the total carrying capacity in the limit. When $k>\left(K_{1} r_{2}\right) /\left(K_{2} r_{1}\right)$ is not too large, the curve increases initially but decreases below zero eventually (dashes and circles). As always, the limiting value for large $D_{i}$ is given by (28). When $k \gg\left(K_{1} r_{2}\right) /\left(K_{2} r_{1}\right)$, the curve has the same shape as for small $k$ : it is monotone decreasing (plot not shown).

\section{Integraldifferenceversusdiffusionrate.png}

Fig. 6 Plot of the integral difference $\int_{\Omega}(u(x)-K(x)) \mathrm{d} x$ versus $D_{1}$ for three values of $k$. The ratio $d=D_{1} / D_{2}$ is fixed at $d=1.1, d=0.9$, and $d=0.5$, corresponding to the dashes and circles, solid, and dashed curves, respectively. Other parameters are the same as in Figure (3).

\section{Discussion}

Spatial population dynamics are often studied via reaction-diffusion equations (Cantrell and Cosner, 2003). There is, however, a certain disconnect: while spatially homogeneous models have found many applications, e.g., for spread rates of biological invasions (Andow et al., 1990; Lewis et al., 2016) or for critical patch sizes in conservation biology (Skellam, 1951), spatially heterogeneous models are largely studied from an abstract analytical point of view. We speculate that among the reasons for this disconnect are that spatially heterogeneous models are almost prohibitively difficult to parametrize since they require too many data points, and also that the available mathematical results are often too abstract and require numerical evaluation. One potential way forward was proposed by Shigesada et al. (1986), who studied persistence and invasions in piecewise constant, periodic landscapes. This simplification not only significantly reduced the number of parameters in the model, it also allowed explicit analytical results that are easy to evaluate and 
apply. Maciel and Lutscher (2013) extended their model framework by introducing individual movement behavior at interfaces between different habitat types, based on earlier work by Ovaskainen and Cornell (2003). Many empirical studies collect the required data (see, e.g., references in Crone et al. (2019)), and the qualitative behavior of the models with interface behavior differs significantly from those without (Maciel and Lutscher, 2013, 2015; Langebrake et al., 2011; Lutscher and Musgrave, 2017; Alqawasmeh and Lutscher, 2019a). However, almost all results on the resulting reaction-diffusion systems with interface matching conditions are based on linear analysis (but see Freedman et al. (1989); Maciel et al. (2019)). Our work here studies several aspects of steady states of these models and applies the results to the question of whether and how the total population density at steady state can exceed the total carrying capacity as a result of movement behavior.

We first establish the existence and uniqueness of steady states by adopting the method of Freedman et al. (1989). We then significantly extent their stability result by proving that this steady state is unconditionally stable. We use the monotonicity results from Maciel et al. (2019) for that. Then we show that the steady-state density is always monotone on each patch: it is either increasing or decreasing on both patches. The density jump at the interface may, however, prevent the solution to be monotone on the entire landscape. We give precise conditions, in terms of the composite parameter $k$, that classify the steady states into one of three cases (Figure 1). We are aware of only one other study of the qualitative behavior of steady states for population dynamics in patchy landscapes: Cruywagen et al. (1996) used a perturbation expansion approach in the model without interface behavior (i.e., with $k=1$ ).

In the critical case where $k=K_{1} / K_{2}$, the steady-state solution is piecewise constant. This case has already shown significance in two ways elsewhere. Langebrake et al. (2011) studied a model for marine reserves and found that the critical value of $k$ delineated the case where model behavior matches empirical results from where it does not; see also Alqawasmeh and Lutscher (2019b). Maciel et al. (2019) studied the evolution of dispersal and patch preference in a two-patch landscape and showed that this special case was an evolutionarily steady strategy and a neighborhood invader strategy.

As an application, we study the question of when the total steady-state density can exceed the total carrying capacity in the landscape. This question has a long tradition in ecology, starting with the work by Freedman and Waltman (1977), who studied it in an ordinary differential equation model of two patches without explicit spatial location. The first treatment of this question with reaction-diffusion equations is by Lou (2006) and later by DeAngelis et al. (2016). A typical result is the existence of a maximum of the total steady-state density at intermediate diffusion rates, and that maximum exceeds the total carrying capacity. Empirical results confirm the existence of such an intermediate maximum (Zhang et al., 2015). For a recent synthesis of this topic and its ecological importance, see Zhang et al. (2017). Our model is the first, based on reaction-diffusion equation, that considers differential movement rates and patch preference in this context. It is also the first that finds that the total density for very large diffusion need not equal the total carrying capacity. Rather, we give precise conditions for when the total density is above or below the total carrying. Arditi et al. (2015) found the same qualitative behavior in a two-patch model with linear dispersal. In fact, their results also include the thresholds $K_{1} / K_{2}$ and $\left(r_{2} K_{1}\right) /\left(r_{1} K_{2}\right)$, but their movement 
behavior does not include bias. We expect that our results can be related to theirs via "patch averaging" (Cobbold and Lutscher, 2014). This is a technique to turn reaction-diffusion systems in patchy landscapes into ordinary differential equation systems with appropriate movement terms between patches.

Arguably the most obvious open question from our work is to find an analytical proof of the numerical and heuristic convergence results for the steady-state density as $D_{1}=d D_{2}$ approach zero and infinity. The discontinuity condition at the interface requires the development of new techniques to prove this convergence. An ecologically more interesting task is to extend this model to more than two patches, each separated by interfaces with corresponding matching conditions, and to study the qualitative behavior of steady states. The infinite periodic case with two patch types is equivalent to our two-patch case, but we are unaware of any study of three or more different patch types.

\section{Appendix}

In this appendix, we provide the Proof of Lemma 5 and a discussion for why we cannot expect a similar result to hold when we choose the ecological (forward Kolmogoroff) diffusion term. The proof is a slight adaptation from Lou (2006). We assume that $u(x)$ is a positive solution of the steady-state equation with Fickian diffusion term and no-flux boundary condition:

$$
\left\{\begin{array}{l}
\frac{\mathrm{d}}{\mathrm{d} x}\left(D(x) \frac{\mathrm{d} u(x)}{\mathrm{d} x}\right)+u(x)(K(x)-u(x))=0, \quad x \in[0, L] \\
D(0) \frac{\mathrm{d} u(0)}{\mathrm{d} x}=0, \quad D(L) \frac{\mathrm{d} u(L)}{\mathrm{d} x}=0 .
\end{array}\right.
$$

We assume that all functions are sufficiently smooth and positive.

First, we divide both sides of the equation by $u(x)$ and integrate by parts over the interval $[0, L]$. After applying the boundary conditions, we obtain:

$$
\begin{aligned}
\int_{0}^{L}(u(x)-K(x)) \mathrm{d} x & =\int_{0}^{L} \frac{1}{u(x)}\left(\frac{\mathrm{d}}{\mathrm{d} x}\left(D(x) \frac{\mathrm{d} u(x)}{\mathrm{d} x}\right)\right) \mathrm{d} x \\
& =\int_{0}^{L} D(x)\left(\frac{1}{u(x)} \frac{\mathrm{d} u(x)}{\mathrm{d} x}\right)^{2} \mathrm{~d} x .
\end{aligned}
$$

Thus, the integral difference is positive, as claimed in the lemma.

Now, we consider the same equation, but with the Fickian diffusion term replaced by the ecological diffusion (or forward Kolmogorov) term (Turchin, 1998):

$$
\begin{cases}\frac{\mathrm{d}^{2}}{\mathrm{~d} x^{2}}(D(x) u(x))+u(x)(K(x)-u(x))=0, & x \in[0, L] ; \\ \left.\frac{\mathrm{d}}{\mathrm{d} x}(D(x) u(x))\right|_{x=0}=0,\left.\quad \frac{\mathrm{d}}{\mathrm{d} x}(D(x) u(x))\right|_{x=L}=0 . & \end{cases}
$$


We follow the same steps as above to find

$$
\begin{aligned}
& \int_{0}^{L}(u(x)-K(x)) \mathrm{d} x=\int_{0}^{L} \frac{1}{u(x)} \frac{\mathrm{d}^{2}}{\mathrm{~d} x^{2}}(D(x) u(x)) \mathrm{d} x \\
& =\int_{0}^{L} \frac{\mathrm{d}}{\mathrm{d} x} D(x)\left(\frac{\mathrm{d}}{\mathrm{d} x} \ln (u(x))\right) \mathrm{d} x+\int_{0}^{L} D(x)\left(\frac{1}{u(x)} \frac{\mathrm{d} u(x)}{\mathrm{d} x}\right)^{2} \mathrm{~d} x .
\end{aligned}
$$

We see that the right-hand side is not necessarily positive. Instead, some conditions on slope or curvature need to be satisfied for positivity. Since the shape of $u$ depends on the shape of $K$ and $D$, it is not obvious what these conditions are.

\section{References}

Y Alqawasmeh and F Lutscher. Movement behaviour of fish, harvesting-induced habitat degradation and the optimal size of marine reserves. Theoretical Ecology, 2019a. 10.1007/s12080-019-0411-x.

Y Alqawasmeh and F Lutscher. Persistence and spread of stage-structured populations in heterogeneous landscapes. Journal of Mathematical Biology, 78(5): 1485-1527, 2019b.

DA Andow, P Kareiva, SA Levin, and A Okubo. Spread of invading organisms. Landscape Ecol., 4(2/3):177-188, 1990.

R Arditi, C Lobry, and T Sari. Is dispersal always beneficial to carrying capacity? New insights from the multi-patch logistic equation. Theoretical Population Biology, 106:45-59, 2015.

RS Cantrell and C Cosner. Spatial ecology via reaction-diffusion equations. Wiley, 2003.

C Cobbold and F Lutscher. Mean occupancy time: linking mechanistic movement models, population dynamics and landscape ecology to population persistence. Journal of Mathematical Biology, 68:549-579, 2014.

F Courchamp, L Berec, and J Gascoinge. Allee effects. Oxford Univ. Press, 2008.

EE Crone, LM Brown, JA Hodgson, F Lutscher, and CB Schultz. Faster movement in nonhabitat matrix promotes range shifts in heterogeneous landscapes. Ecology, 10, 2019. 10.1002/ecy.2701.

GC Cruywagen, P Kareiva, MA Lewis, and JD Murray. Competition in a spatially heterogeneous environment: Modelling the risk of spread of a genetically engineered population. Theoretical Population Biology, 49(1):1-38, 1996.

D DeAngelis, W-M Ni, and B Zhang. Dispersal spatial heterogeneity: single species. Journal of Mathematical Biology, 72(1):239-254, 2016. /10.1007/s00285-015-0879-y.

HI Freedman. Deterministic mathematical models in population ecology. Marcel Dekker, New York, 1980.

HI Freedman and P Waltman. Mathematical models of population interactions with dispersal. I: stability of two habitats with and without a predator. SIAM Journal on Applied Mathematics, 32(3):631-648, 1977.

HI Freedman, JB Shukla, and Y Takeuchi. Population diffusion in a two patch environment. Mathematical Biosciences, 95(1):111-123, 1989.

J Langebrake, L Riotte-Lambert, CW Osenberg, and P De Leenheer. Differential movement and movement bias models for marine protected areas. Journal of Mathematical Biology, 2011. 
MA Lewis, SV Petrovskii, and JR Potts. The mathematics behind biological invasions, volume 44. Springer, 2016.

ou(2006)Lou:2006:EMS Y Lou. On the effect of migration and spatial heterogeneity on single and multiple species. Journal of Differential Equations, 223(2): 400-426, 2006.

F Lutscher and J Musgrave. Behavioral responses to resource heterogeneity can accelerate biological invasions. Ecology, 98(5):1229-1238, 2017.

F Lutscher, MA Lewis, and E McCauley. The effects of heterogeneity on population persistence and invasion in rivers. Bulletin of Mathematical Biology, 68(8):21292160, 2006.

G Maciel, C Cosner, RS Cantrell, and F Lutscher. Evolutionary stable movement strategies in reaction-diffusion models with edge behavior. Journal of Mathematical Biology, 2019. 10.1007/s00285-019-01339-2.

GA Maciel and F Lutscher. How individual movement response to habitat edge effects population persistence and spatial spread. The American Naturalist, 182 (1):42-52, 2013.

GA Maciel and F Lutscher. Allee effects and population spread in patchy landscapes. Journal of Biological Dynamics, 9(1):109-123, 2015.

O Ovaskainen and S Cornell. Biased movement at a boundary and conditional occupancy times for diffusion processes. Journal of Applied Probability, 40(3): 557-580, 2003.

SW Pacala and J Roughgarden. Spatial heterogeneity and interspecific competition. Theoretical Population Biology, 21:92-113, 1982.

L Perko. Differential equations and dynamical systems. Springer, New York, 2001.

N Shigesada, K Kawasaki, and E Teramoto. Traveling periodic waves in heterogeneous environments. Theoretical Population Biology, 30:143-160, 1986.

JG Skellam. Random dispersal in theoretical populations. Biometrika, 38:196-218, 1951.

P Turchin. Quantitative analysis of movement. Sinauer Associates, Sunderland, 1998.

B Yurk and C Cobbold. Homogenization techniques for population dynamics in strongly heterogeneous landscapes. Journal of Biological Dynamics, 12:171-193, 2018.

B Zhang, X Liu, DL DeAngelis, W-M Ni, and G Geoff Wang. The effect of dispersal on total biomass in a patchy, heterogeneity system: analysis and experiment. Mathematical Biosciences, 264:54-62, 2015.

B Zhang, A Kula, K Mack, L Zhai, A Ryce, W-M Ni, D DeAngelis, and JD Van Dyken. Carrying capacity in a heterogeneous environment with habitat connectivity. Ecology letters, 20(9):1118-1128, 2017. 